\title{
SMITH-LEMLI-OPITZ SYNDROME. CASE REPORT
}

\author{
Sorin Ioan Iurian ${ }^{1,2}$, Livia Ognean ${ }^{3}$, Han Brunner ${ }^{4}$, Leo Kluijtmans ${ }^{5}$, Petr Jira ${ }^{6}$, \\ Dana Fintina ${ }^{2}$, Bogdan Ionut Mehedintu ${ }^{2}$ \\ 1 "Lucian Blaga" University, Sibiu \\ ${ }^{2}$ Pediatric Clinic Hospital, Sibiu \\ ${ }^{3}$ Neonatology Clinic, Emergency Clinical Hospital, Sibiu \\ ${ }^{4}$ Medical Genetics Department, Radboud University, Nijmegen, The Nederlands \\ ${ }^{5}$ Translational Metabolic Laboratory, Nijmegen, The Nederlands \\ ${ }^{6}$ Jreoen Bosch Hospital, Pediatric Department, The Nederlands
}

\begin{abstract}
Smith-Lemli-Opitz syndrome (SLOS) is an autosomal-recessive inherited disease characterized by multiple anomalies secondary to cholesterol synthesis impairment. The authors present diagnosis peculiarities and limitation of treatment options in a 5-weeks old infant with ambiguous genitalia.
\end{abstract}

Keywords: Smith-Lemli-Opitz syndrome, infant, hypocholesterolemia

\section{INTRODUCTION}

Smith-Lemli-Opitz syndrome (SLOS) is an autosomal-recessive inherited disease characterized by multiple anomalies secondary to cholesterol synthesis impairment. The syndrome was initially described by Smith, Lemli and Opitz in 1964 (1).

Etiology. The disease cause remained unknown until 1993 when Irons and al (2) remarked, for SLOS patients, low plasma cholesterol levels and increased serum for sterol precursors such as 7-dehydrocholesterol (7DHC) and 8-dehydrocholesterol (8DHC). The syndrome is caused by deficiency of the enzyme 7-dehydrocholesterol-reductase (DHCR7), the enzyme involved in 7DHC conversion to cholesterol.

Pathophysiology. The consequence of enzyme deficit is accumulation of cholestorol precursors and plasma cholesterol reduction. The cholesterol is involved in synthesis of cell membranes, steroid hormones (Fig. 1), bile acids and myelin. There is an impaired connection between cholesterol and embryonic protein "Shh" (during first gestational week) affecting the development of face, heart, limbs, brain.

\section{Epidemiology}

The disease prevalence was estimated to be between 1:20.000 - 1:60.000 births among caucasians and sex ratio is $1: 1$.

\section{Mortality and morbidity}

The rate of spontaneous abortions for SLOS foetuses is high. The death rate is also high, especially during first week of life, due to multiple system failure. Cholesterolemia under $20 \mathrm{mg} \%$ represents bad prognosis in term of survival. SLOS patients can also exhibit hepatopathy (3), hearing loss, impaired visual acuity (cataract) (4) and failure to grow and develop. The neonates can exhibit intrauterine growth restriction, severe hypotonia and a peculiar cry (shrill).

Previous studies performed by Kelley and Hennekam (2000), based on analysis of 164 biochemically confirmed SLOS cases, showed the following

Coresponding author:

Sorin loan lurian, „Lucian Blaga“ University, 2-4 Pompeiu Onofreiu Street, Sibiu

E-mail: iurian_sorsab@hotmail.com 
Cholesterol

$3 \beta$ hydroxy-steroid dehydrogenase (3ßHSD)
$17 \alpha$ hydroxylase

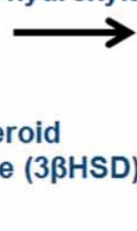

Progesterone

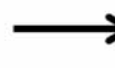

17-OH

Progesterone

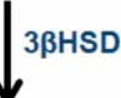 \\ 17-OH \\ Pregnenolon}

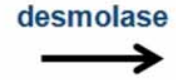

Dehydroepiandrosterone (DHEA)

3BHSD

desmolase

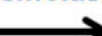


Androstendione

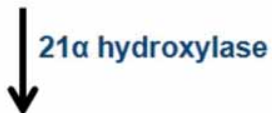

$\downarrow 21 \alpha$ hydroxylase

Deoxycorticosterone

11 Deoxycortisol
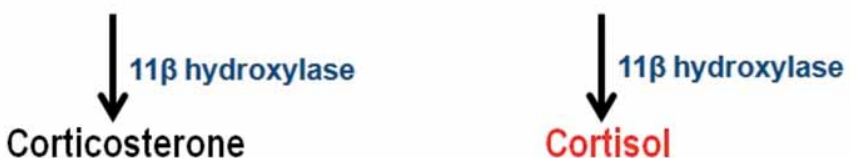

Corticosterone

Cortisol

18 hydroxylase

18 hydro-steroid

dehydrogenase

Aldosterone

FIGURE 1. Enzimatic pathways of hormones synthesis from cholesterol

anomalies: mental retardation (95\% of cases), postpartum growth failure (82\%), microcephaly (84\%), structural brain anomalies (37\%), palpebral ptosis $(70 \%)$, cataract $(22 \%)$, anteverted nostrils $(78 \%)$; cleft palate $(47 \%)$, congenital heart defects $(54 \%)$, abnormal lung lobulation (45\%), piloric stenosis $(14 \%)$, colonic aganglionosis $(16 \%)$, kidney anomalies $(43 \%)$, genital anomalies $(65 \%), 2 / 3$ toes syndactyly (97\%) and postaxial polydactyly (48\%). Disease severity is variable: from subtle disabilities to severely affected patients.

\section{SLOS diagnosis}

Prenatally, illness suspicion is based on ultrasound exam of foetus revealing suggestive anomalies and justifying evaluation of cholesterol precursors in amniotic fluid and 7DHCR enzyme activity in chorionic villous. The genetic test of 7DHCR gene is another diagnosis possibility. Triple or quadruple screen markers from maternal serum suggestive for Down syndrome correlated with normal karyotype could represent a diagnosis marker for SSLO. Shackleton (5) demonstrated estriol presence in mothers' urine carrying SLOS foetuses, allowing prenatal screening.
Postnatally, the diagnosis is biochemically or genetically confirmed. Biochemical studies imply low plasma cholesterol, striking elevation for serum $7 \mathrm{DHC}$ and for ratio between cholesterol precursors/plasma cholesterol. Evaluation of cholesterolemia alone isn't recommended. Imagistic studies of brain, heart and abdominal organs can detect SLOS anomalies. Brainstem-evoked response identifies hearing loss.

\section{Treatment}

There is no efficient therapy for SLOS patients (6). We mention several therapeutic possibilities:

- oral cholesterol supplementation could increase plasma cholesterol due to feed-back inhibition of hydroxy-methyl-glutaryl-coenzyme A - reductase (HMG-CoA reductase). Cholesterol recommended daily dose is $100 \mathrm{mg} / \mathrm{kg}$. body weight, using sources like egg yolk, sour cream or butter. Studies confirmed lower absorption of crystalline cholesterol in suspension as compare to its absorption from egg yolk (7). Because cholesterol isn't able to pass into the central nervous system, there is no behaviour improvement for SLOS patients with oral cholesterol supplementation $(8,9)$; 
- oral bile acids supplementation with doubtful efficiency;

- statins (eg. Simvastatin) can decrease the ratio precursors/cholesterol through 2 mechanisms:

1. HMG-CoA reductase blockage reduces cholesterol precursors' levels;

2. activation of 7DHC reductase (enzyme "upregulation") improves cholesterolemia (10).

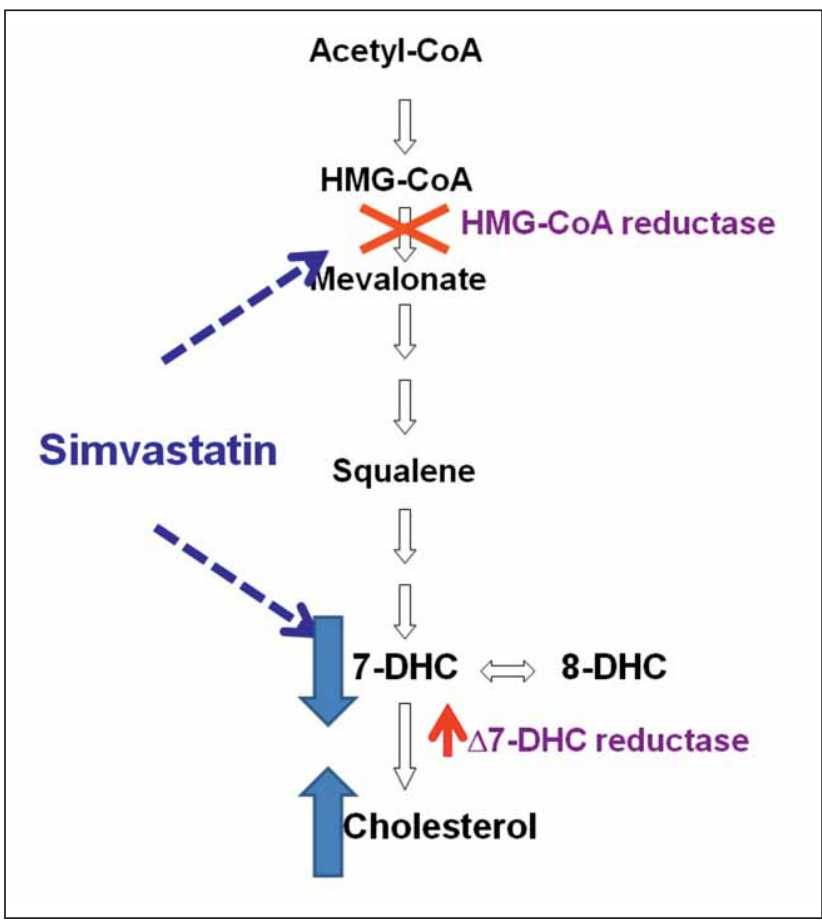

FIGURE 2. Simvastatin: action mechanism (according to Petr Jira).

Surgical therapy could implies: polydactyly repair, gastrostomy placement, cleft palate repair, pyloromyotomy or surgical management for Hirschsprung disease.

\section{CASE PRESENTATION}

T.B., 5 week-old infant, is transferred from the neonatology clinic for evaluation due to genital ambiguity, plurimalformative syndrome and craniofacial dysmorphism.

Family history: healthy and non- consanguineous parents; mother II Gesta I Para (first pregnancy-miscarriage). Obstetric history: birth at 9 months gestational age through caesarean at 19 hours after spontaneous rupture of amniotic membranes, meconial amniotic fluid; 3,010 g weight; APGAR score $=7 / 1 \mathrm{~min}$; incorrect sucking reflex has determined nasogastric feeding tube insertion.

Investigations (neonatology department) revealed: polyglobulia, hyponatremia and hyperkalemia (salt wasting syndrome?), hypocholesterol- emia (37 mg\%). Genital ambiguity justified karyotyping which confirmed male gender $(46, X Y)$. Imagistic evaluation revealed corpus callosum hypoplasia, adrenal glands with disorganized structure and identified testicles at scrotal level. The unsatisfactory weight curve led to addition of hipercaloric milk via nasogastric tube.

The clinical exam (pediatric clinic): weight 3,000 g, microcephaly; pale skin; bilateral palmar simian crease; impaired nutritional status, generalized hypotonia, cranio-facial dysmorphism (down slanted palpebral fissures, anteverted nostrils, micrognathia; posteriorly rotated ears), bilateral syndactyly affecting toes $2 / 3$, genital ambiguity (micropenis, bifid scrotum, scrotal/penile hypospadias).

Investigations: $\mathrm{WBC}=22,700 / \mathrm{mm}^{3}(\mathrm{Ly}=48 \%)$, $\mathrm{Hgb}=10.54 \mathrm{~g} \%$; Plt $=243,000 / \mathrm{mm}^{3}$; hepatic and renal markers within normal range; tendencies to hyponatremia and hyperpotassemia (with improvement in evolution); hypocholesterolemia $(27 \mathrm{mg} \%$ ). Endocrinological assessment: 17-hydroxyprogesterone $=7.51 \mathrm{ng} / \mathrm{ml}$ (normal 3.6-13.7); cortisolemia $=16.3 \mu \mathrm{g} / \mathrm{dl}$ (normal 15-25); DHEA (dehydroepiandrosterone) $=2.37 \mathrm{ng} / \mathrm{ml}$ (normal 0.26-3.85); testosteronemia $=0.16 \mathrm{ng} / \mathrm{ml}$ (normal $1,42-9,21)$; aldosteronemia $=28.4 \mathrm{ng} / \mathrm{dl}$ (normal 1.76-23.2 ng/dl).

Abdominal ultrasound exam showed left kidney duplication with hydronephrosis and the auditory function testing confirmed unilateral hearing loss.

In context of genital ambiguity correlated with karyotype, the differential included:

- masculine undervirilisation due to testicular differentiation disorders and/or androgen disorders (insensitivity to androgens and 5 - $\alpha$-reductase deficiency syndrome); these entities are considered unlikely (see hypotestosteronemia);

- 21-hydroxylase deficiency with hypervirilisation has been excluded based on 46,XY karyotype and normal serum values for 17-hydroxyprogesterone/DHEA;

- disorders of sex chromosome differentiation (Turner and Klinefelter syndromes, mixed gonadal dysgenesis, true hermaphroditism) based on karyotype $46, \mathrm{XY}$;

- syndromes involving genital ambiguity (Denis-Drash, Goldenhar, WAGR, Robinow, SLOS) have also been analyzed.

\section{Positive diagnosis}

In context of plurimalformative syndrome (cranio-facial dysmorphism, genitourinary malformations, brain abnormalities) in conjunction with hy- 
pocholesterolemia, authors considered SLOS as highly probable, justifying sterolic profile testing that revealed high values for 7DHC $(113 \mu \mathrm{mol} / 1$, normal $<5 \mu \mathrm{mol} / \mathrm{l})$ and for $8 \mathrm{DHC}(83 \mu \mathrm{mol} / 1$, normal $<1 \mu \mathrm{mol} / \mathrm{l})$ and hypocholesterolemia (614 $\mu \mathrm{mol} / 1$, normal 2,000-5,200). Thus, authors biochemically confirmed SLOS (genetic test isn't required). The case is regarded as type II of disease (see multiple associated malformations).

The treatment options implied:

1. Adequate nutritional support (on nasogastric tube) consisting of breast milk and high calorie nutrient; oral intake of cholesterol (1 yolk egg/day);

2. Improvement of precursors/cholesterol ratio by starting Simvastatin therapy $(1 \mathrm{mg} / \mathrm{kg}$. body weight daily).

\section{Evolution}

On the $9^{\text {th }}$ day of hospitalization, the infant developed septic condition justifying initiation of broad-spectrum antibiotics with favourable evolution. Authors remarked persistence of microcephaly and growth impairment despite nutritional interventions. Biologically, authors noticed increasing of cholesterolemia (from $27 \mathrm{mg} \%$ to $39 \mathrm{mg} \%$ ) without significant neurological improvement.

\section{CONCLUSIONS}

1. The authors focused on an infant exhibiting the plurimalformative syndrome and severe hypocholesterolemia, the latter detail leading diagnosing process towards SLOS;

2. Special mention should be made of the specific endocrinological profile, given its low testosterone levels and normal values of cortisolemia and aldosteronemia, although cholesterol represents the synthesis substrate for all these hormones. Hormone substitution therapy (testosterone) isn't necessary. Authors mentioned transient evolution of salt wasting syndrome;

3. Since first pregnancy ended in abortion during first trimester, the embryo damage due to SLOS is considered highly probable.;

4. In evolution, the case requires the monitoring of therapeutic effects, testing the sterolic profile once a trimester (under Simvastatin therapy), reconstruction of external genitalia corresponding to male phenotype, auditory prosthesis and psychological support. Thus, therapy is complex, requiring many specialists intervention (paediatrician, psychologist, audiologist, endocrinologist, surgeon, neurologist etc).

\section{REFERENCES}

1. Smith D.W., Lemli L., Opitz J.M. A newly recognized syndrome of multiple congenital anomalies. J Pediatr. 1964;64:210-7.

2. Irons M., Elias E.R., Tint G.S. Abnormal cholesterol metabolism in the Smith-Lemli-Opitz syndrome: report of clinical and biochemical findings in four patients and treatment in one patient. Am J Med Genet. 1994; 50(4):347-52.

3. Rossi M., Vajro P., lorio R., et al. Characterization of liver involvement in defects of cholesterol biosynthesis: long-term follow-up and review. Am J Med Genet A. 2005; 132(2):144-51.

4. Goodwin H., Brooks B.P., Porter F.D. Acute postnatal cataract formation in Smith-Lemli-Opitz syndrome. Am J Med Genet A. 2008;146A(2):208-11.

5. Shackleton C.H., Roitman E., Kratz L.E. Equine type estrogens produced by a pregnant woman carrying a Smith- Lemli-Opitz syndrome fetus. J Clin Endocrinol Metab. 1999; 84(3):1157-9.

6. Svoboda M.D., Christie J.M., Eroglu Y. at al. Treatment of Smith-Lemli-Opitz syndrome and other sterol disorders. Am J Med Genet C Semin Med Genet. 2012; 160C(4):285-94.

7. Lin D.S., Steiner R.D., Flavell D.P. et al. Intestinal absorption of cholesterol by patients with Smith-Lemli-Opitz syndrome, Pediatr Res. 2005, 57:765-70.

8. Tierney E., Conley S.K., Goodwin H. et al. Analysis of short-term behavioral effects of dietary cholesterol supplementation in SmithLemli-Opitz syndrome. Am J Med Genet A. 2010; 152A(1):91-5.

9. Sikora D.M., Ruggiero M., Petit-Kekel K. et al. Cholesterol supplementation does not improve developmental progress in Smith-Lemli-Opitz syndrome. J Pediatr 2004; 144: 783-791.

10. Jira P.E., Wevers R.A., de Jong J. et al. Simvastatin. A new therapeutic approach for Smith-Lemli-Opitz syndrome. J Lipid Res 2000; 41: 1339-1346. 